\title{
Recognizing nitrogen dopant atoms in graphene using atomic force microscopy
}

\author{
Nadine J. van der Heijden, ${ }^{1}$ Daniël Smith, ${ }^{1}$ Gaetano Calogero, ${ }^{1,2}$ Rik S. Koster, ${ }^{3}$ Daniel Vanmaekelbergh, ${ }^{1}$ \\ Marijn A. van Huis, ${ }^{3}$ and Ingmar Swart ${ }^{1}$ \\ ${ }^{1}$ Condensed Matter and Interfaces, Debye Institute for Nanomaterials Science, Utrecht University, \\ P.O. Box 80 000, 3508 TA Utrecht, The Netherlands \\ ${ }^{2}$ DTU Natotech, Technical University of Denmark, Building 345E, rsteds Plads, DK-2800 Kongens Lyngby, Denmark \\ ${ }^{3}$ Soft Condensed Matter, Debye Institute for Nanomaterials Science, Utrecht University, P.O. Box 80 000, 3508 TA Utrecht, The Netherlands
}

(Received 15 April 2016; revised manuscript received 7 June 2016; published 27 June 2016)

\begin{abstract}
Doping graphene by heteroatoms such as nitrogen presents an attractive route to control the position of the Fermi level in the material. We prepared $\mathrm{N}$-doped graphene on $\mathrm{Cu}(111)$ and $\operatorname{Ir}(111)$ surfaces via chemical vapor deposition of two different molecules. Using scanning tunneling microscopy images as a benchmark, we show that the position of the dopant atoms can be determined using atomic force microscopy. Specifically, the frequency shift-distance curves $\Delta f(z)$ acquired above a $\mathrm{N}$ atom are significantly different from the curves measured over a $\mathrm{C}$ atom. Similar behavior was found for $\mathrm{N}$-doped graphene on $\mathrm{Cu}(111)$ and $\operatorname{Ir}(111)$. The results are corroborated by density functional theory calculations employing a van der Waals functional.
\end{abstract}

DOI: 10.1103/PhysRevB.93.245430

\section{INTRODUCTION}

The position of the Fermi level in conventional semiconductors can be controlled by introducing dopant atoms into the lattice. The same approach can be used for graphene [1-5]. In order to understand the effect of dopant atoms in graphene, it is essential to be able to study the number of dopants, their distribution, and how they are incorporated in the lattice, ideally down to the atomic level.

Scanning tunneling microscopy (STM) has been used to study the geometric and electronic structure of mechanically exfoliated and epitaxially grown graphene [6-15] and graphene nanostructures [16-21]. Doped graphene has also been studied with STM. For example, it was found that nitrogen- and boron-dopant atoms in graphene have a characteristic appearance in STM images, allowing their identification [22-24]. Furthermore, it was found that the nitrogen atoms in graphene, grown by chemical vapor deposition, occupy predominantly one sublattice of graphene [25]. Due to the convolution of geometric and electronic contributions to the STM signal, it is typically not straightforward to determine how the dopant atoms are incorporated into the lattice. However, precisely the incorporation in the lattice determines how the dopant atoms affect the properties of the host material [26].

Atomic force microscopy (AFM) can image the geometric structure of graphite and graphene with atomic resolution [3,27-34]. Chemical recognition of atoms in a material with AFM is however nontrivial. It has been shown that AFM-based force-distance spectroscopy can provide chemical contrast between the chemically very different atoms $\mathrm{Pb}, \mathrm{Si}$, and $\mathrm{Sn}$ in a surface alloy [35]. More recently, the chemical reactivity of boron- and nitrogen-doped graphene grown on silicon carbide was investigated with AFM [24]. Chemically passivated tips can also provide different contrast above boron atoms in graphene [5]. Since we aim to identify two chemical elements that are similar in size and are expected to have the same coordination in the lattice, we opted to use metal tips. Metal tips are not chemically inert and we expected that by using metal tips discerning small differences would be easier than with functionalized tips.
Here, we describe a method by which individual N-dopant atoms in graphene can be recognized by AFM. Using STM data as a benchmark, we show that the minima in frequency shift-distance $[\Delta f(z)]$ spectra are distinctly different for $\mathrm{N}$ and $\mathrm{C}$ atoms. This behavior was observed for graphene grown on $\mathrm{Cu}(111)$ and $\operatorname{Ir}(111)$ surfaces. By exploiting the well-defined moiré pattern of the latter, we analyzed the influence of height corrugation of the substrate, as well as the coupling strength to the surface. The experimental results are corroborated by density functional theory (DFT) calculations.

\section{METHODS}

\section{A. Sample preparation and synthesis}

Clean $\mathrm{Cu}(111)$ and $\operatorname{Ir}(111)$ crystals were prepared using several sputtering (with argon) and annealing cycles. For the synthesis of $\mathrm{N}$-doped graphene on $\mathrm{Cu}(111)$, a protocol from Zabet-Khosousi et al. was adapted [25], while for synthesis on $\operatorname{Ir}(111)$ we adapted the method described by NDiaye et al. [7]. Two types of precursor molecules were used: 1,10phenanthroline (Phen) and 1,2,4-triazolo(1,5-a)pyrimidine (Pyr), both purchased from Sigma-Aldrich and used without further purification (indicated purity: 99\% for Phen and 99\% for Pyr). Their chemical structures are shown in Figs. 1(a) and 1(b), respectively. Note that the ratio between $\mathrm{N}$ and $\mathrm{C}$ atoms in the molecules is 1:6 for Phen and 4:5 for Pyr. Precursors were thermally evaporated onto a hot $\mathrm{Cu}(111)\left(875^{\circ} \mathrm{C}\right)$ or $\operatorname{Ir}(111)$ $\left(1200^{\circ} \mathrm{C}\right)$ surface. After molecular deposition, the temperature of the metal crystals was kept constant at these temperatures for 20 minutes for $\mathrm{Cu}(111)$ and for 30 seconds for $\operatorname{Ir}(111)$. The $\operatorname{Ir}(111)$ crystal was transferred out of the preparation chamber at an elevated temperature to minimize the adsorption of residual molecules onto the surface.

\section{B. Experimental procedures}

The experiments were performed using a combined LT STM/AFM from Scienta Omicron GmbH. The base pressure was lower than $2 \times 10^{-9} \mathrm{mbar}$, and the working temperature was $4.6 \mathrm{~K}$. A commercially available qPlus sensor with a 
(a)<smiles>c1cnc2c(c1)ccc1cccnc12</smiles>

(b)<smiles>c1ccn2ncnc2c1</smiles>

FIG. 1. (a) 1,10-phenanthroline (Phen). (b) 1,2,4-triazolo[1,5a]pyrimidine (Pyr).

resonance frequency $f_{0} \approx 25 \mathrm{kHz}$, a spring constant $k \approx$ $1800 \mathrm{~N} / \mathrm{m}$, and a quality factor $Q \approx 25 \mathrm{k}$ was used, which was operated with a peak-to-peak amplitude of approximately $2 \AA$ A All STM images were obtained in constant-current mode, with the bias applied to the sample. All AFM images were acquired in constant-height mode. 3D frequency shift $(\Delta f)$ data were obtained by taking multiple constant-height AFM images at stepwise increasing sample-tip distance (z). Semiactive drift compensation was applied by correlating STM images obtained between AFM images to determine the lateral drift and compensating for this between consecutive AFM images. Tips providing atomic resolution were prepared by controlled contact with the metal surface and voltage pulses, resulting in a sharp metal tip apex. The tip apex was not functionalized because we expect that using a chemically active tip apex would enable discerning small differences in chemical properties of the investigated atoms. From the 3D data cube, we extracted the coordinates of the minima of all $\Delta f(z)$ curves $\left(\Delta f_{\min }\right.$ and $\left.z_{\min }\right)$ by fitting a parabolic function to points up to $0.5 \mathrm{~Hz}$ above the most negative $\Delta f$ value.

\section{Density functional theory calculations}

Density functional theory (DFT) simulations were performed on a crystallographic model of graphene/Ir(111). The hexagonal supercell consisted of a $(9 \times 9 \times 4)$ slab of iridium atoms and a single $(10 \times 10)$ layer of graphene; see Fig. 2 [10]. The calculations employed the projector augmented plane wave method [36-38] and the generalized gradient approximation (GGA) and the exchange-correlation functional formulated by Perdew, Burke, and Ernzerhof (PBE) [39] as implemented in the Vienna $A b$ initio Simulation Package (VASP) [37,40,41]. Van der Waals forces were added by DFT-D3 (BJ damping) [42], and a $\Gamma$-centered $(3 \times 3 \times 1)$ $k$-point mesh was used for sampling. Cutoff values for the wave functions and augmentation functions of $400 \mathrm{eV}$ and $560 \mathrm{eV}$ were used, respectively. Table I gives some key numbers concerning the corrugation of the graphene layer with respect to the $\operatorname{Ir}(111)$ substrate, as well as a comparison to previous work. Our results are in good agreement with previously reported values. We found an adsorption energy of $88 \mathrm{meV}$ per $\mathrm{C}$ atom, which is close to previously reported values [10].

$\Delta f(z)$ curves were simulated based on calculations involving a tetrahedral metal $\operatorname{Ir}(111)$ cluster of four atoms that was brought closer to the sample in a stepwise manner. The size of the tip cluster was limited to four atoms (in two layers) to balance the computational cost (considering the large unit cell needed to describe the substrate) with the accuracy (we used small oscillation amplitudes, increasing the sensitivity to short-range chemical forces). However, our calculations will underestimate the magnitude of the electrostatic contribution to the total tip-sample force [43]. During these calculations the tip-apex atom, the probed atom, and its three nearest neighbors were allowed to relax their position; the positions of all other atoms of the graphene and iridium slab were fixed. We fitted the as-obtained data points with a Morse potential. The fitted energy-distance curves were first converted to force-distance curves by taking the derivative with respect to distance, and subsequently to $\Delta f(z)$ curves using the method described by Giessibl [44].

\section{RESULTS AND DISCUSSION}

We first describe the results for $\mathrm{N}$-doped graphene on $\mathrm{Cu}(111)$. Figures 3(a) and 3(b) show an overview and higher magnification image of a graphene film grown using Phen. The
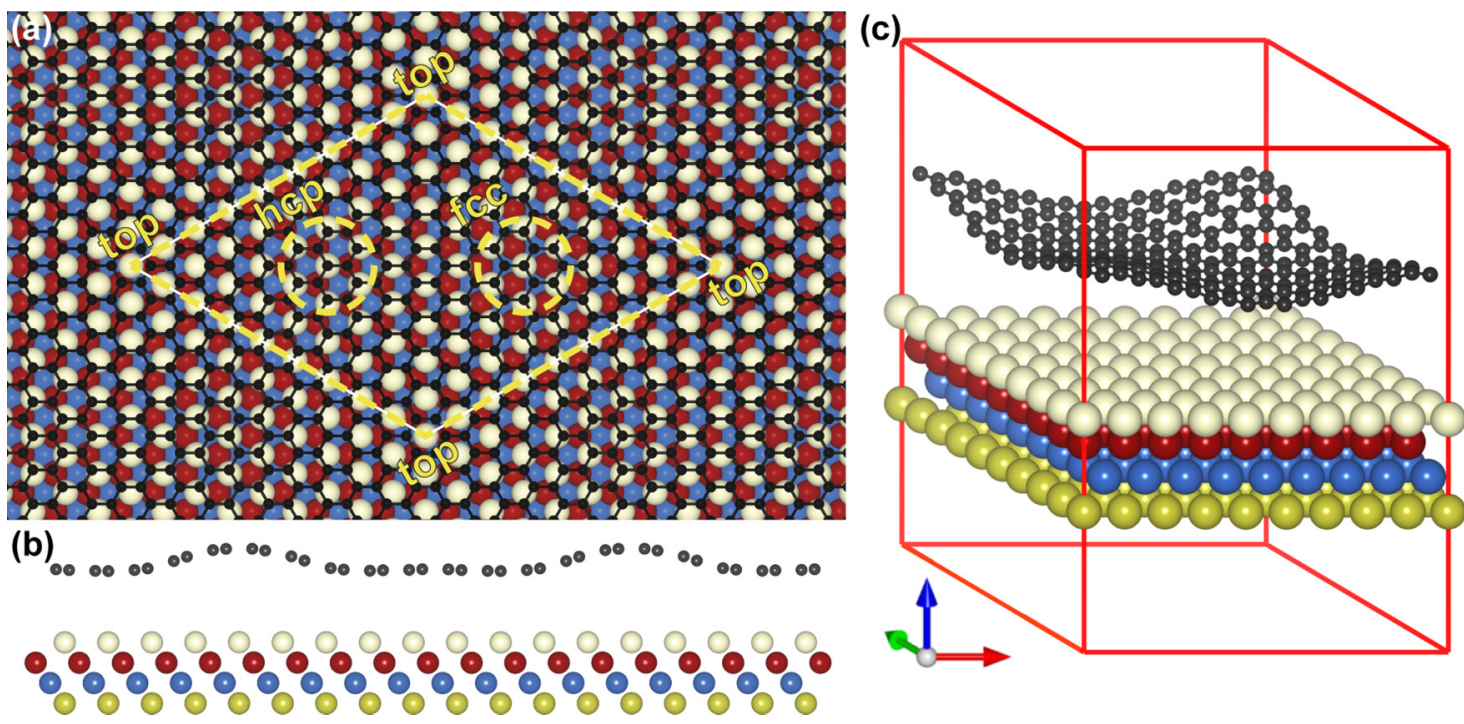

FIG. 2. Crystallographic model of graphene/Ir(111). Carbon atoms are indicated in black. Iridium atoms are colored by layer. Top, second, third, and bottom layers are colored white, red, blue, and yellow, respectively. (a) Top view, with the supercell and its characteristic positions outlined in yellow. (b) Side view; height differences in graphene corrugation are amplified by a factor of 10. (c) Oblique projection. 
TABLE I. Corrugation of the graphene moiré pattern on $\operatorname{Ir}(111)$. The value of $h$ in the various positions is computed using the height of the nearest Ir atom as a reference.

\begin{tabular}{lccccccc}
\hline \hline Reference & Method & $h_{\text {top }}(\AA)$ & $h_{\text {fcc }}(\AA)$ & $h_{\text {hcp }}(\AA)$ & $h_{\text {bridge }}(\AA)$ & $\bar{h}(\AA)$ & $\Delta h(\AA)$ \\
\hline Hämäläinen et al. [34] & LEED & 3.71 & 3.29 & 3.27 & n/a & $3.39 \pm 0.03$ & $0.43 \pm 0.09$ \\
Busse et al. [10] & DFT & 3.62 & 3.29 & 3.27 & n/a & 3.41 & 0.35 \\
Voloshina et al. $[33]$ & DFT & 3.58 & 3.28 & 3.27 & 3.315 & $\mathrm{n} / \mathrm{a}$ & $\mathrm{n} / \mathrm{a}$ \\
This work & DFT & $3.63^{\mathrm{a}}$ & $3.32^{\mathrm{b}}$ & 3.31 & 3.32 & 3.40 & 0.34 \\
\hline \hline
\end{tabular}

${ }^{\mathrm{a}}$ All the atoms that constitute the hexagon in the top position have the same height.

${ }^{\mathrm{b}}$ The values for $h_{\mathrm{fcc}}, h_{\mathrm{hcp}}$, and $h_{\text {bridge }}$ correspond to the height of the lowest atom among otherwise equivalent sites.

overview image shows that synthesis of $\mathrm{N}$-doped graphene on $\mathrm{Cu}(111)$ resulted in flaked, irregular, small patches of graphene
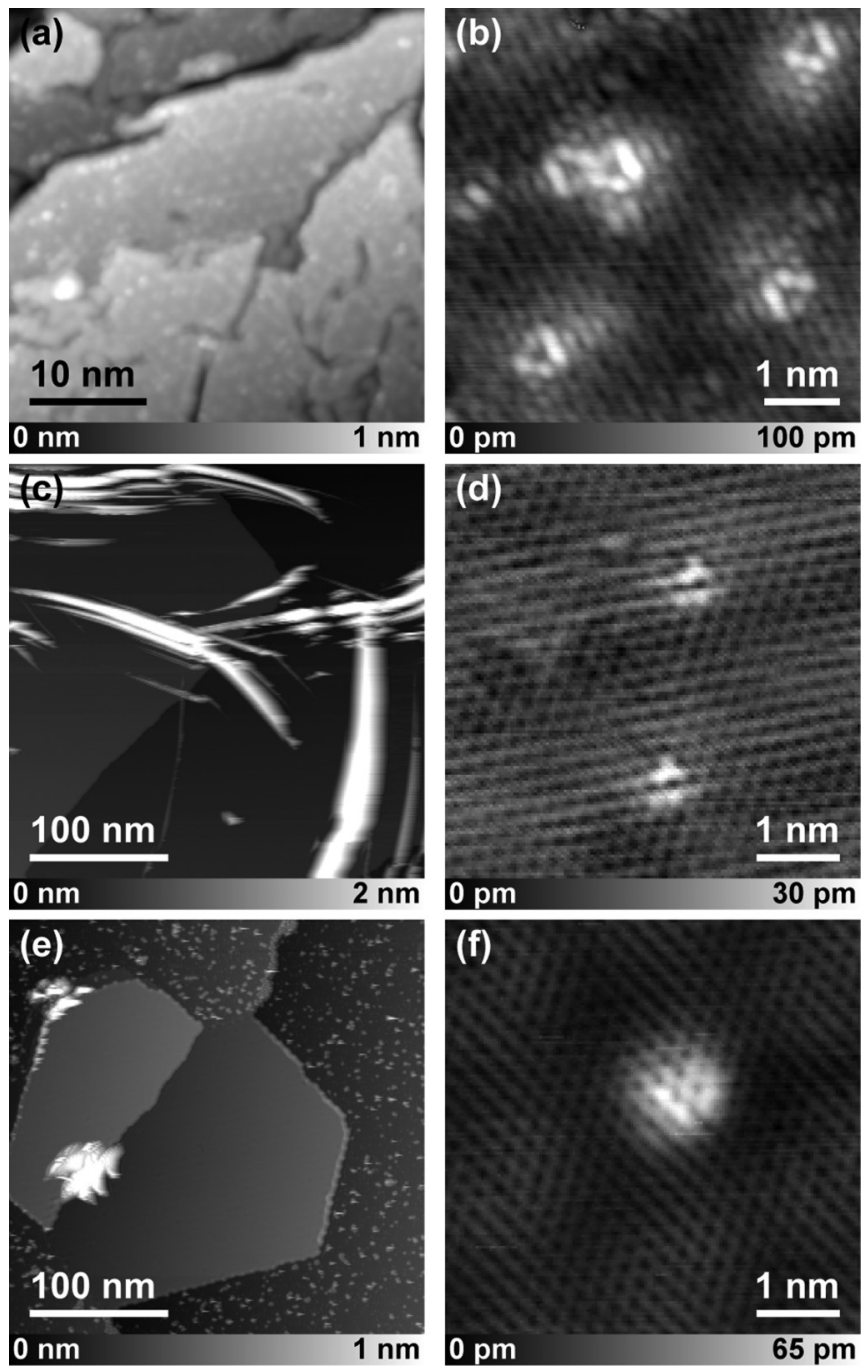

FIG. 3. (a), (b) Overview and zoom of graphene on $\mathrm{Cu}(111)$ grown with Phen precursor molecules. (a) STM set point $20 \mathrm{pA}$ at $100 \mathrm{mV}$. (b) STM set point $50 \mathrm{pA}$ at $1.0 \mathrm{~V}$. Dopant concentration approximately $1 \%$. (c), (d) Overview and zoom of graphene on $\operatorname{Ir}(111)$ grown with Phen precursor molecules. (c) STM set point $1 \mathrm{nA}$ at 100 $\mathrm{mV}$. (d) STM set point $1 \mathrm{nA}$ at $100 \mathrm{mV}$. Dopant concentration less than $1 \%$. (e), (f) Overview and zoom of graphene on $\operatorname{Ir}(111)$ grown with Pyr precursor molecules. (e) STM set point $1 \mathrm{nA}$ at $100 \mathrm{mV}$. (f) STM set point $500 \mathrm{pA}$ at $100 \mathrm{mV}$. Dopant concentration less than $1 \%$. with a diameter up to $100 \mathrm{~nm}$. A more zoomed view of such a graphene patch, Fig. 3(b), shows characteristic triangularshaped features that have been assigned to individual $\mathrm{N}$ dopants in graphene $[23,25,45]$. Determining the optimal parameters to grow large graphene flakes on $\mathrm{Cu}(111)$ using the method described above is beyond the scope of the present paper.

Phen can also be used to grow N-doped graphene on $\operatorname{Ir}(111)$. In this case, films with lateral sizes on the order of micrometers were obtained. Figure 3(c) shows an STM image of such a large graphene sheet. The elongated features with larger apparent height, i.e., the white stripes, are due to rippling of the graphene sheet $[6,46]$. Higher magnification STM images of the graphene films on $\operatorname{Ir}(111)$ also feature triangular shapes, not present in pristine graphene; see Fig. 3(d). These features have previously been identified as signatures of $\mathrm{N}$ dopants [45].

As evidenced by the STM images shown in Figs. 3(e) and 3(f), graphene films on $\operatorname{Ir}(111)$ could also be grown using Pyr as the precursor molecule. Despite the fact that this molecule has a significantly higher $\mathrm{N}$ to $\mathrm{C}$ ratio, the concentration of dopant atoms was found to be of the same order of magnitude as that of the graphene sheets grown with Phen. In the films grown with Pyr molecules, we encountered triangular-shaped features with a significantly larger spatial extension, as shown in Fig. 3(f). Similar features have previously been attributed to two $\mathrm{N}$ atoms in close proximity $[22,23]$.

The fact that the $\mathrm{N}$ dopants have a well-defined signature in STM images means that we can use this data to benchmark the AFM data; i.e., by sequentially acquiring an STM and AFM image of the same area, the location of the $\mathrm{N}$ atom in the graphene can be pinpointed. Figures 4(a) and 4(b) show an STM and AFM image of the same area of a N-doped graphene film on $\mathrm{Cu}(111)$. Again, the STM image shows a clear feature due to the $\mathrm{N}$ atom. However, it is not possible to determine the location of the $\mathrm{N}$ atom directly from the STM or the AFM images. The STM image is not atomically resolved at the location of and in the immediate surrounding of the dopant atom. In contrast, the six-membered rings of the graphene lattice are clearly resolved in the AFM image. Interestingly, in the vicinity of the $\mathrm{N}$ atom, the six-membered rings appear heavily distorted. From DFT calculations the lateral stretching in the graphene network induced by the substitutional dopant is only about $1 \%$ in compression to the nearest-neighbor distance. Thus these distortions are imaging artifacts and are most likely due to the electrostatic interaction between the dopant atom, which has a partial positive charge, and the dipole moment of the tip $[42,43,47,48]$. A Bader charge 

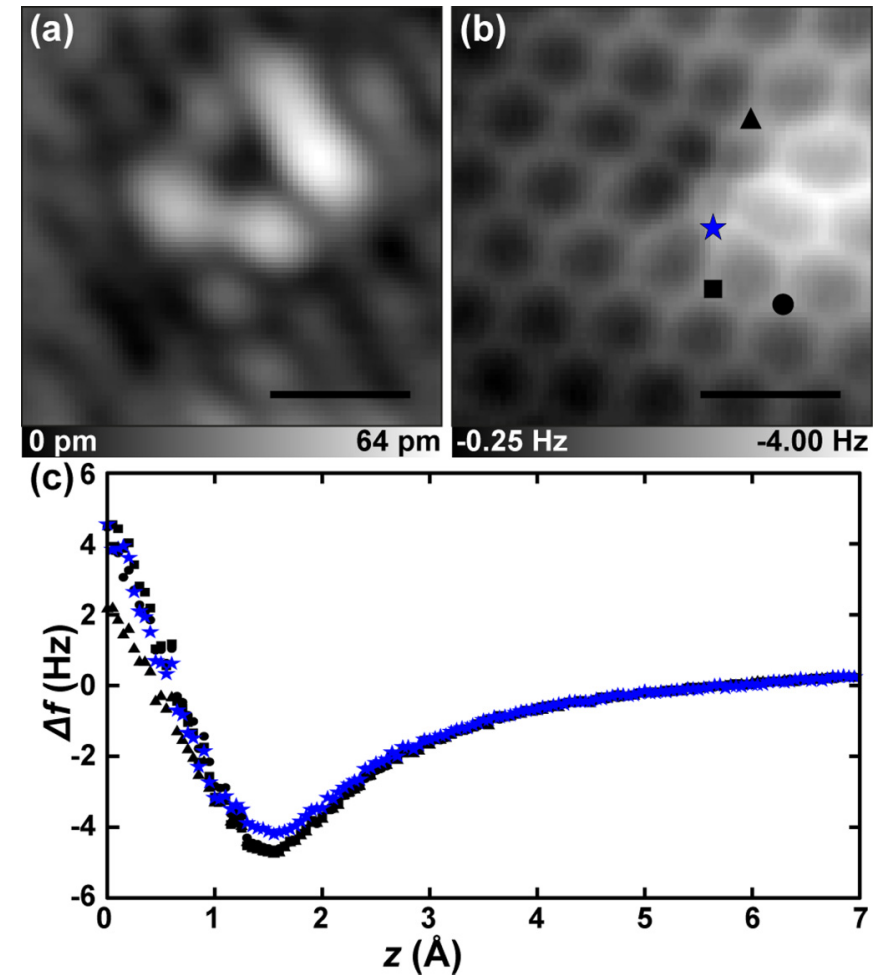

FIG. 4. (a) STM image of a $\mathrm{N}$ dopant in graphene on $\mathrm{Cu}(111)$, set point $50 \mathrm{pA}$ at $1.0 \mathrm{~V}$. (b) Constant-height AFM image of the same location at $-20 \mathrm{pm}$ with respect to STM set point. (c) $\Delta f(z)$ curves obtained over the positions as indicated in (b). Scale bars are $5 \AA$.

analysis shows that the $\mathrm{N}$ atom has a charge of $1.27 e$, where $73 \%$ of that charge is extracted from the 3 neighboring $\mathrm{C}$ atoms donating $0.3 e$ each to the $\mathrm{N}$ atom. The proposed location of the $\mathrm{N}$ atom in the AFM image, indicated by the blue star marker in Fig. 4(b), is not at the center of the triangular signature of the STM image, despite the fact that the two images, Figs. 4(a) and 4(b), are obtained over exactly the same location. Due to tip geometry the location where the signature currents are observed can be slightly offset with respect to the location of the lattice sites as measured by AFM. The region of less negative frequency shift values at the right-hand side of the image is due to the vertical corrugation of the film [33,49].

We acquired several $\Delta f(z)$ curves at atoms that should have (approximately) the same height above the surface; see the markers in Fig. 4(b). The spectra are shown in Fig. 4(c). Note that the spectra on three equivalent $\mathrm{C}$ atoms overlap, whereas the spectrum taken above the $\mathrm{N}$ atom is significantly different. In particular, the minimum of the $\Delta f(z)$ curve over an $\mathrm{N}$ atom is less negative than over a $\mathrm{C}$ atom at an equivalent position in the lattice. This trend was observed for different tips. This demonstrates that the method used by Sugimoto et al. [35], where AFM can be used to discriminate atoms, can be successfully extended to $\mathrm{N}$ and $\mathrm{C}$ atoms in graphene on $\mathrm{Cu}(111)$.

To investigate the effect of the dopant on the potential-energy landscape as probed by the AFM, a $\Delta f$ data cube was acquired by taking a series of constant-height AFM images at increasing tip-sample distance. From this data cube, a $\Delta f(z)$ spectrum and therefore the coordinates of the minimum, $\Delta f_{\min }$ and $z_{\min }$, can be extracted for every pixel [30].
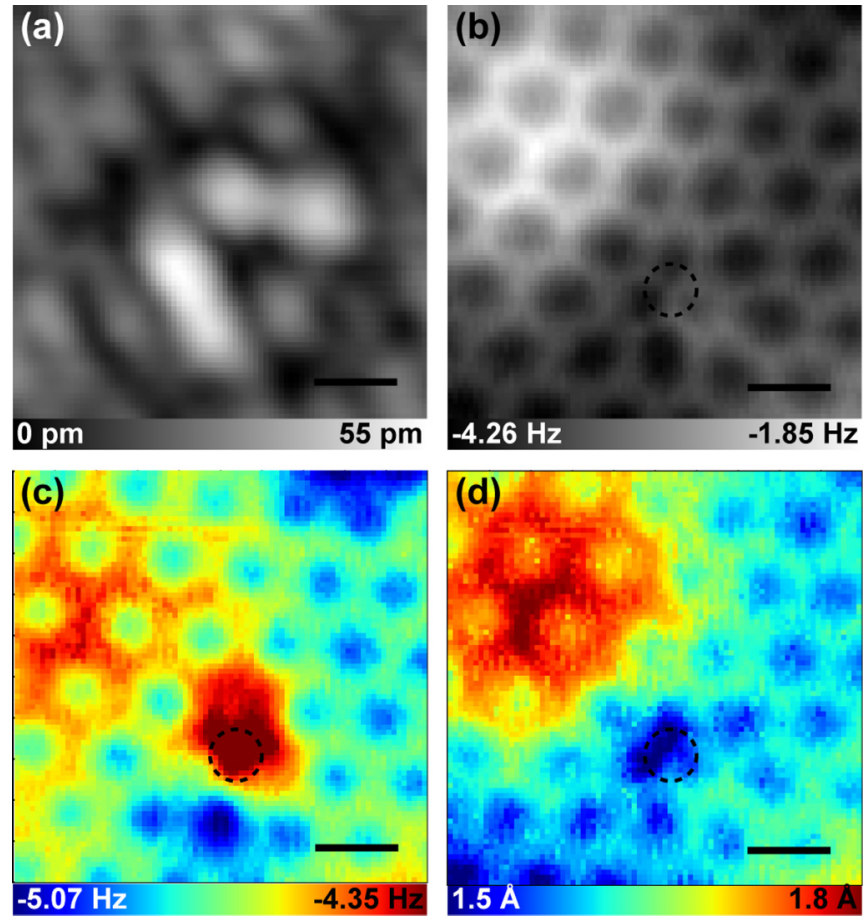

FIG. 5. (a) STM image of a $\mathrm{N}$ dopant in graphene on $\mathrm{Cu}(111)$, set point $50 \mathrm{pA}$ at $1.0 \mathrm{~V}$. (b) Constant-height AFM image of the same location at $-20 \mathrm{pm}$ with respect to $\mathrm{STM}$ set point. (c) $\Delta f_{\min }$ map of the same area. (d) Corresponding $z_{\min }$ map, where 0 corresponds to -20 pm with respect to STM set point. Scale bars are $3 \AA$.

Figures 5(a) and 5(b) show STM and AFM images obtained over the same location. In the STM image, the characteristic feature of a N dopant is observed, while in the AFM image sixmembered rings can be seen. Again, there are image distortions close to the $\mathrm{N}$ dopant. The $\Delta f_{\min }$ and $z_{\min }$ maps extracted from a $\Delta f$ data cube are shown in Figs. 5(c) and 5(d), respectively. From the $\Delta f_{\min }$ map it is clear that the effect of the $\mathrm{N}$ atom on the minimum $\Delta f$ follows the same trend as before: the minimum $\Delta f$ above the $\mathrm{N}$ atom is generally less negative than over $\mathrm{C}$ atoms. This effect is not localized on a single atom. This is attributed to the influence of the partially charged $\mathrm{N}$ atom on the charge density of the atoms to which it is bound [24]. At the location of the dopant atom there is also some contrast in the $z_{\min }$ map. In particular, the tip has to approach the $\mathrm{N}$ atom more closely to reach the minimum of the $\Delta f(z)$ curve.

The $\Delta f_{\min }$ and $z_{\min }$ maps exhibit a larger region of contrast (less negative frequency shift and larger tip-sample distance). As can be seen by comparing the maps to the constant-height AFM image, this region coincides with a top site of the moiré pattern. The fact that the geometric structure of the sample influences the $\Delta f_{\min }$ map can be rationalized as follows: due to the corrugation of the graphene, the van der Waals (vdW) attraction between the bulk of the tip and the graphene differs between top and valley sites; i.e., in a valley site the tip will experience a larger $\mathrm{vdW}$ attraction from the surrounding top sites, while at a top site the surrounding valleys are farther away, resulting in a smaller vdW attraction.

To investigate the general applicability of this approach and to study the influence of the sample-substrate interaction, we 
performed the same experiments on $\mathrm{N}$-doped graphene grown on an $\operatorname{Ir}(111)$ surface. Graphene on $\operatorname{Ir}(111)$ is physisorbed, but exhibits a chemical modulation [10]. The $\mathrm{C}$ atoms located at top sites of the moiré pattern have a weak vdW interaction with the substrate, while the atoms in the hcp site form a weak covalent bond to the surface. This provides the opportunity to investigate the influence of the interaction between the surface and the species of interest on the chemical recognition by AFM.

First we present $\mathrm{N}$-doped graphene on $\operatorname{Ir}(111)$ made with precursor Phen, Figs. 6(a)-6(d). A unit cell of the moiré lattice is overlaid on the images to indicate the positions of the dopant atoms relative to their position in the moiré pattern. From the STM image, Fig. 6(a), we can see that there are two $\mathrm{N}$ dopants at approximately the same lattice positions with respect to the moiré pattern, namely an hcp site. Consequently, they have a similar effect on the surrounding energy landscape. In the AFM image, Fig. 6(b), the top sites appear elongated towards the positions of the dopants. The elongation effect cannot be caused by an asymmetry of the tip as top sites that do not have a $\mathrm{N}$ atom in an adjacent hcp site (right and left side of the image) do not show the elongation. A similar elongation is found in the $\Delta f_{\min }$ and $z_{\min }$ maps, Figs. 6(c) and 6(d). As was observed for $\mathrm{N}$-doped graphene on $\mathrm{Cu}(111), \Delta f_{\min }$ is significantly less negative over the $\mathrm{N}$ atom than over $\mathrm{C}$ atoms located at the same position in the moiré unit cell.

If we overlay the moiré lattice extracted from the constantheight AFM image onto the $\Delta f_{\min }$ and $z_{\min }$ maps, there is a significant mismatch for the former; i.e., the locations of least negative frequency shift appear shifted with respect to the positions of the top sites of the moiré lattice. In contrast, the moire lattice extracted from the AFM image fits the $z_{\text {min }}$ map which was extracted from the same data as the $\Delta f_{\min }$ map quite well. The shift in the $\Delta f_{\text {min }}$ map is most likely caused by an asymmetry in the mesoscopic tip shape. If the tip is asymmetric at this length scale, the vdW attraction will also be asymmetric. The same argumentation, used to explain why the corrugation of the surface is visible in the $\Delta f_{\min }$ map, explains why the moiré top positions appear shifted in the $\Delta f_{\text {min }}$ map. Important to note here is that only the apparent positions of the top and valley sites have changed: the actual moiré pattern positions, as well as the locations of the dopant atoms, remain the same. The characteristic contrast of the $\mathrm{N}$ atoms is still at their hcp locations in the overlaid moire grid, where the $\Delta f_{\min }$ for $\mathrm{N}$ is less negative than for the surrounding $\mathrm{C}$ atoms.

Figures 6(e)-6(h) show results from a set of experiments performed on $\mathrm{N}$-doped graphene grown using Pyr. Interestingly, the STM signature is very different from that shown in Fig. 6(a) and has a significantly larger spatial extension. From the AFM image, it is concluded that the feature observed in the STM channel is located at the top site of the moire lattice. Previously, a very similar signature in STM images was attributed to two $\mathrm{N}$ atoms on neighboring positions on the same sublattice, i.e., with one $\mathrm{N}$ atom in the meta position with respect to the other [23]. Figures $6(\mathrm{~g})$ and $6(\mathrm{~h})$ show the $\Delta f_{\min }$ and $z_{\min }$ maps. Also in this case, the area where the $\mathrm{N}$ atoms are expected to be has a clearly different signature in both maps compared to C-only top sites of the moiré lattice. Note that the difference between the top sites in the $z_{\text {min }}$ map is much larger than typical variations observed between identical top sites
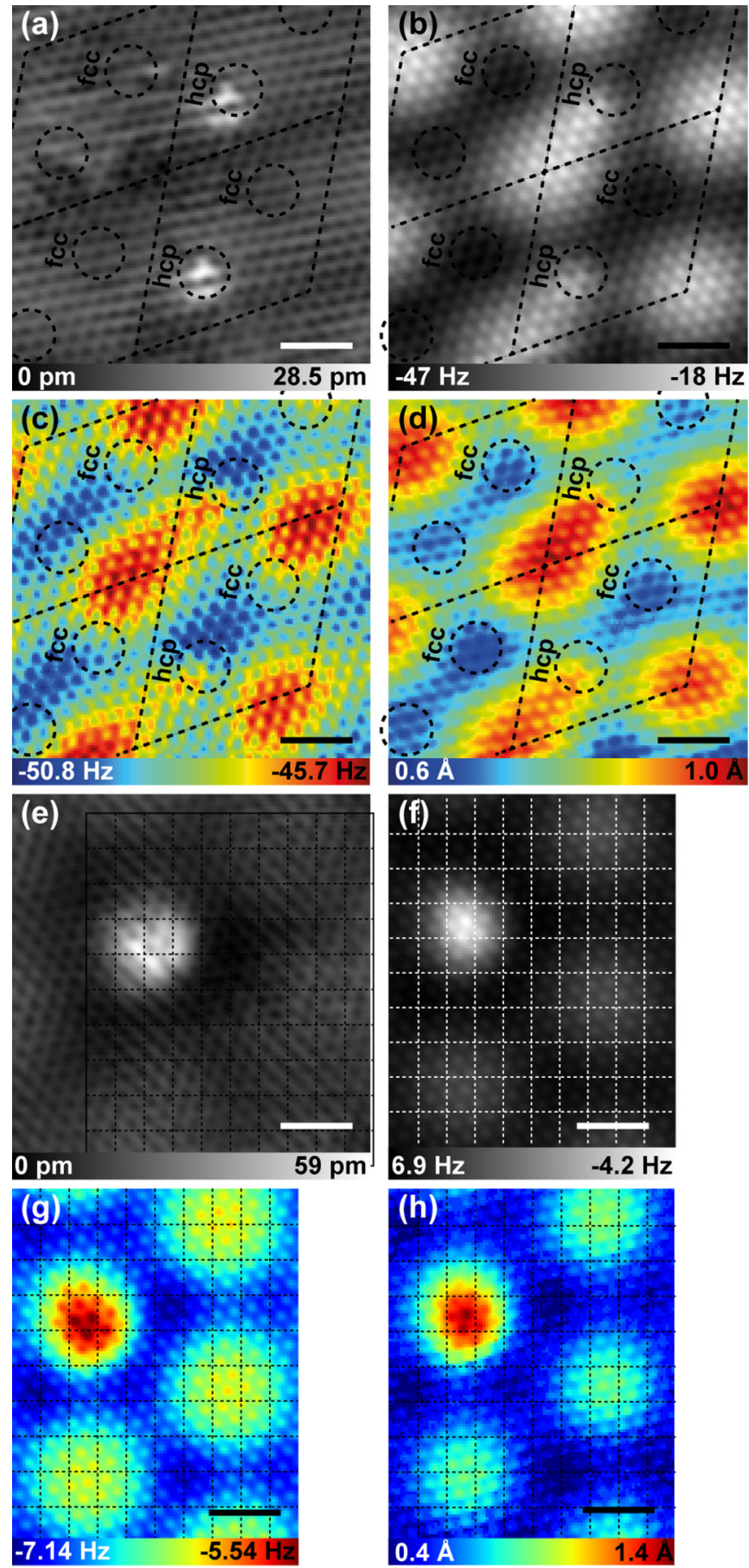

FIG. 6. (a) STM image of a $\mathrm{N}$ dopant in graphene on $\operatorname{Ir}(111)$ grown with Phen, at set point $1 \mathrm{nA}$ at $100 \mathrm{mV}$. (b) Constant-height AFM image of the same location at $-100 \mathrm{pm}$ with respect to STM set point. (c) $\Delta f_{\min }$ map of the same area. (d) Corresponding $z_{\min }$ map. (e) STM image of a N dopant in graphene on $\operatorname{Ir}(111)$ grown with Pyr, at set point $500 \mathrm{pA}$ at $100 \mathrm{mV}$. (f) Constant-height AFM image of the same location at $-150 \mathrm{pm}$ with respect to STM set point. (g) $\Delta f_{\min }$ map of the same area. (h) Corresponding $z_{\min }$ map. Scale bars are $1 \mathrm{~nm}$.

[34], reinforcing the assignment that the feature observed in the STM image is due to incorporation of (multiple) $\mathrm{N}$ atoms at that site. 
The contrast over the rings/atoms is reversed in Figs. 6(b)-6(d) in comparison to Figs. 6(f)-6(h). That is, in Figs. 6(b)-6(d), the centers of the hexagonal rings are more attractive (larger negative frequency shift) than the apparent bonds, whereas this trend is reversed in Figs. 6(f)-6(h). The contrast inversion in constant-height AFM images of graphene has been reported before and is due to the offsets in the $\Delta f(z)$ curves acquired over apparent bonds and the center of the hexagons [15].

To corroborate the experimental findings, we performed DFT calculations. In particular, we simulated $\Delta f(z)$ curves obtained for $\mathrm{N}$-doped graphene on $\operatorname{Ir}(111)$. We chose to model graphene on $\operatorname{Ir}(111)$ because of its well-defined moiré pattern that can be modeled well by a periodic supercell, taking $(10 \times 10)$ unit cells of graphene on $(9 \times 9)$ cells of $\operatorname{Ir}(111)$ $[10,33]$. We simulated $\Delta f(z)$ curves for $\mathrm{N}$ and $\mathrm{C}$ atoms in two distinctive positions in the moiré pattern. These are the top and hcp sites of the moiré unit cell, as indicated in Fig. 2(a). At the top sites, the interaction between graphene and $\operatorname{Ir}(111)$ is mainly due to vdW interactions, whereas at the hcp sites a weak covalent bond is formed between $\mathrm{C}$ atoms and the underlying Ir atoms [10]. The results of the energy-distance calculations, as well as the corresponding fits to a Morse potential, are shown in Figs. 7(a) and 7(b). The blue curves are for a $\mathrm{N}$ atom, the black curves for a $\mathrm{C}$ atom in the same position. From these curves we calculated the corresponding $\Delta f(z)$ curves, Figs. 7(c) and 7(d).

For both the top and hep sites, the simulated $\Delta f(z)$ curves are clearly distinct for $\mathrm{N}$ and $\mathrm{C} ; \Delta f_{\text {min }}$ is significantly less negative above $\mathrm{N}$ compared to $\mathrm{C}$, in qualitative agreement with our experimental observations for $\mathrm{N}$-doped graphene on both $\mathrm{Cu}(111)$ and $\operatorname{Ir}(111)$. The value of $\Delta f_{\min , \mathrm{C}}-\Delta f_{\min , \mathrm{N}}$ differs between simulation and experiments and also between the experiments, being approximately $10 \mathrm{~Hz}, 0.5 \mathrm{~Hz}, 3 \mathrm{~Hz}$, and $1 \mathrm{~Hz}$ for the simulation and the $\mathrm{Cu}(111)$ and two $\operatorname{Ir}(111)$ experiments, respectively. The fact that the experimental values for the difference between $\mathrm{N}$ and $\mathrm{C}$ (data acquired with different tips) are not identical points to the importance of the chemical composition and geometry of the tip apex. As stated earlier, we only used one tip geometry and composition in the DFT calculations. It is likely that the electrostatic contribution is underestimated [43]. Furthermore, uncertainties in the experimental parameters needed to convert the calculated $E(z)$ curves to $\Delta f(z)$ curves contributes to the observed discrepancy between the experimental and simulated values of the frequency shift. Because of the above, we only focus on qualitative trends in the DFT results.

Note that the $z$ position of the minimum is shifted towards the surface (smaller $z$ value) for the hcp site, in agreement with the vertical corrugation of the moiré pattern. These findings imply that the surface-graphene interaction has a negligible influence on the chemical recognition of $\mathrm{N}$ dopants in graphene on $\operatorname{Ir}(111)$.

The same trend is found in the simulations and in the copper and iridium experiments, which is a good indication that the observed trend $\left(\Delta f_{\min }\right.$ is less negative for a $\mathrm{N}$ atom than for a $\mathrm{C}$ atom) is robust, regardless of location or substrate. We should note that Telychko et al. found the reverse relationship [24]; however their experiments employed a silicon carbide surface instead of the metallic surfaces presented in this paper.
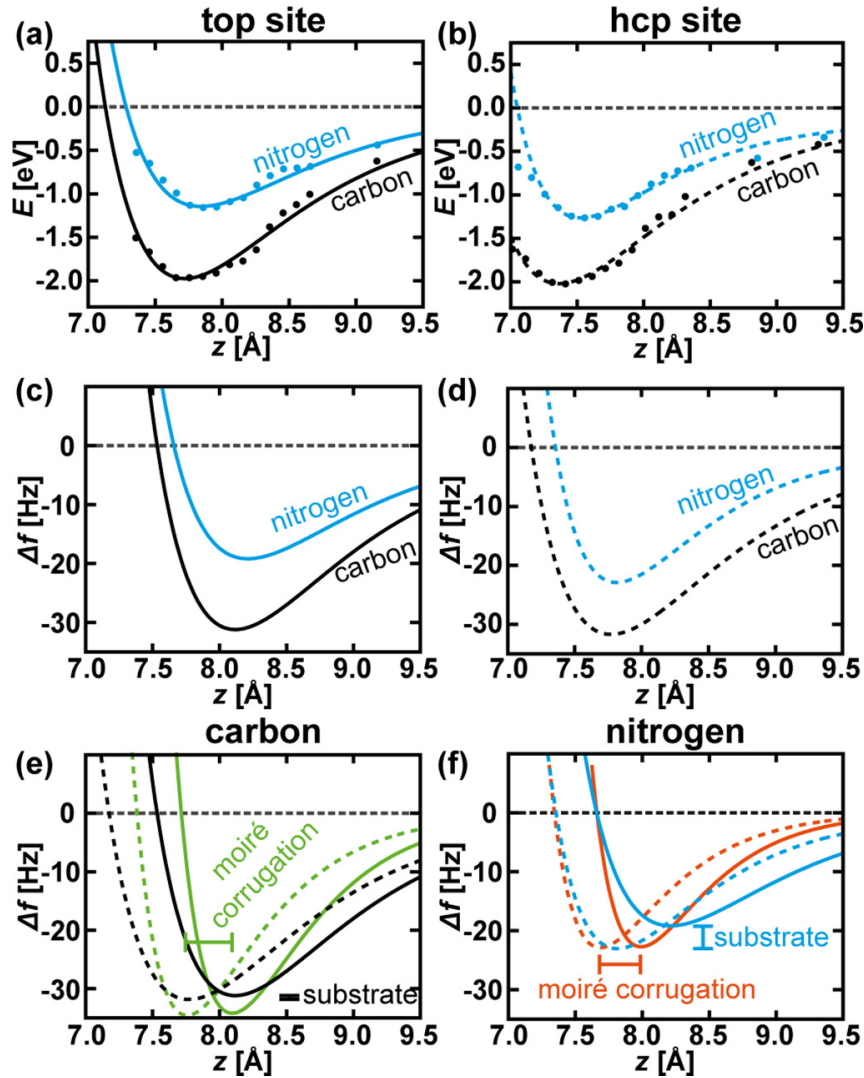

FIG. 7. Energy-distance DFT calculations for N-doped graphene on $\operatorname{Ir}(111) . z$ is the vertical distance between the average height of the atoms in the topmost Ir layer in the $\operatorname{Ir}(111)$ substrate and the height of the topmost Ir atoms of the tip model. (a) Comparison between $\mathrm{N}$ and $\mathrm{C}$ at a top site. (b) Comparison between $\mathrm{N}$ and $\mathrm{C}$ at an hep site. (c) $\Delta f(z)$ curves calculated from the energy-distance curves comparing $\mathrm{N}$ and $\mathrm{C}$ at a top site. (d) $\Delta f(z)$ curves calculated from the energydistance curves comparing $\mathrm{N}$ and $\mathrm{C}$ at an hcp site. (e) Comparison between $\mathrm{C}$ atoms at top (solid lines) and hcp (dashed lines) sites, with $\operatorname{Ir}(111)$ substrate (black) and without $\operatorname{Ir}(111)$ substrate (green). (f) Comparison between $\mathrm{N}$ atoms at top (solid lines) and hcp (dashed lines) sites, with $\operatorname{Ir}(111)$ substrate (blue) and without $\operatorname{Ir}(111)$ substrate (orange).

We theoretically investigated the effect of the substrate. This was done by performing similar calculations to those described above using a graphene sheet without underlying substrate, but having the same moire corrugation as in the case of the simulations including the $\operatorname{Ir}(111)$ substrate, and comparing the results to those obtained for the calculations including the substrate. The resulting curves for the $\mathrm{C}$ atom are shown in Fig. 7(e). Black lines correspond to the results obtained from calculations including the substrate [these are reproduced from Figs. 7(c) and 7(d)], whereas green lines are the results obtained from the simulations without the substrate. Figure 7 (f) shows the same, but for the $\mathrm{N}$ atom, where the blue lines are for calculations that include the $\operatorname{Ir}(111)$ substrate [these are reproduced from Figs. 7(c) and 7(d)] and the orange curves are calculated without substrate. The solid lines correspond to spectra over atoms at a top site, while the dashed lines correspond to $\Delta f(z)$ curves simulated over an hcp site. 
TABLE II. Corrugation of the N-doped graphene moiré pattern on $\operatorname{Ir}(111)$ for different dopant positions. Bold numbers indicate that the atom considered in that specific high-symmetry position is a nitrogen.

\begin{tabular}{lcccccc}
\hline \hline N position & $\Delta h_{\text {Ir }}(\AA)$ & $h_{\text {top }}(\AA)$ & $h_{\text {fcc }}(\AA)$ & $h_{\text {hcp }}(\AA)$ & $h_{\text {bridge }}(\AA)$ & $\bar{h}(\AA)$ \\
\hline pristine & 0.027 & 3.633 & 3.318 & 3.311 & 3.323 & 3.403 \\
N at top & 0.031 & $\mathbf{3 . 6 3 3}$ & 3.323 & 3.311 & 3.326 & 3.401 \\
N at fcc & 0.047 & 3.629 & $\mathbf{3 . 3 8 3}$ & 3.321 & 3.327 & 3.405 \\
N at hcp & 0.048 & 3.630 & 3.330 & $\mathbf{3 . 3 7 4}$ & 3.334 & 3.405 \\
N at bridge & 0.042 & 3.630 & 3.331 & 3.334 & $\mathbf{3 . 3 8 2}$ & 3.404 \\
\hline \hline
\end{tabular}

First, we will discuss the results obtained for C, Fig. 7(e). For the calculation including substrate, the $z$ position of the minimum for $\mathrm{C}$ atoms in hcp and top sites is shifted to smaller $z$ (compare dashed and solid black lines). As can be inferred from Table I, the difference in $z$ between top and hcp sites is approximately $0.32 \AA$. This corresponds very well to the shift in $z$ of the minimum (indicated by the green bar). Removing the substrate from the calculation does not affect the position of the minimum in $z$ (compare black and green lines). This implies that the position of the minimum reflects the corrugation of the sample.

The depth of the minimum, i.e., $\Delta f_{\min }$, is virtually identical for top and hcp sites for simulations with (compare solid and dashed black lines) and without the $\operatorname{Ir}(111)$ (compare solid and dashed green lines). The difference in $\Delta f_{\min }$ in the calculations (black bar) is much smaller than what is found experimentally. This is attributed to the underestimation of the $\mathrm{vdW}$ contribution by the small $\mathrm{Ir}_{4}$ cluster used as the model for the tip in the DFT calculation. In the calculations the additional $\mathrm{vdW}$ attraction caused by the bulk tip was only accounted for by a homogeneous offset. Note that the absolute value for $\Delta f_{\text {min }}$ does differ for calculations with (black) and without (green) substrate. This offset is due to the vdW interaction between the tip and the Ir atoms of the substrate. From this result, we infer that the interaction between the tip and a $\mathrm{C}$ atom in graphene/ $\operatorname{Ir}(111)$ is (almost) unaffected by the interaction between graphene and the $\operatorname{Ir}(111)$.

Next, we turn our attention to the $\mathrm{N}$ atom, Fig. 7(f). Again, the moiré corrugation results in a shift of $z_{\text {min }}$ between top (solid) and hcp (dashed) sites, indicated by the orange bar. This is observed for calculations with (blue) and without (orange) substrate. Table II gives key heights for the moiré corrugation of graphene with $\mathrm{N}$ dopant atoms at various sites. From this table it can be seen that the moiré corrugation is hardly disturbed by the dopant atoms. $\mathrm{N}$ dopants at top sites appear at the same site as their $\mathrm{C}$ counterparts in pristine graphene. For the other three sites we see that the $\mathrm{N}$ dopants protrude a few pm with respect to the original position of $\mathrm{C}$ in pristine graphene. Furthermore, the $\mathrm{C}-\mathrm{N}$ distances are virtually identical to the $\mathrm{C}-\mathrm{C}$ distances ( $1 \%$ contraction). Hence, we ascribe the shift in $z_{\min }$ between top (solid) and hcp (dashed) lines to the moiré corrugation.

Now focus on $\Delta f_{\min }$ for the calculation without substrate (orange curves). The depth of the minimum, i.e., $\Delta f_{\min }$, is the same for atoms in hep and top sites. This is consistent with the fact that in the absence of the substrate, the $\mathrm{N}$ atoms in the different sites are chemically equivalent and have equal surroundings. As established for $\mathrm{C}$ above, the vertical corrugation of the graphene itself leads to only a very small change in the depth of the minimum. Turning to the calculation including the substrate (blue lines), the $\Delta f_{\min }$ is significantly less negative for the top site (solid blue), than for the hcp site
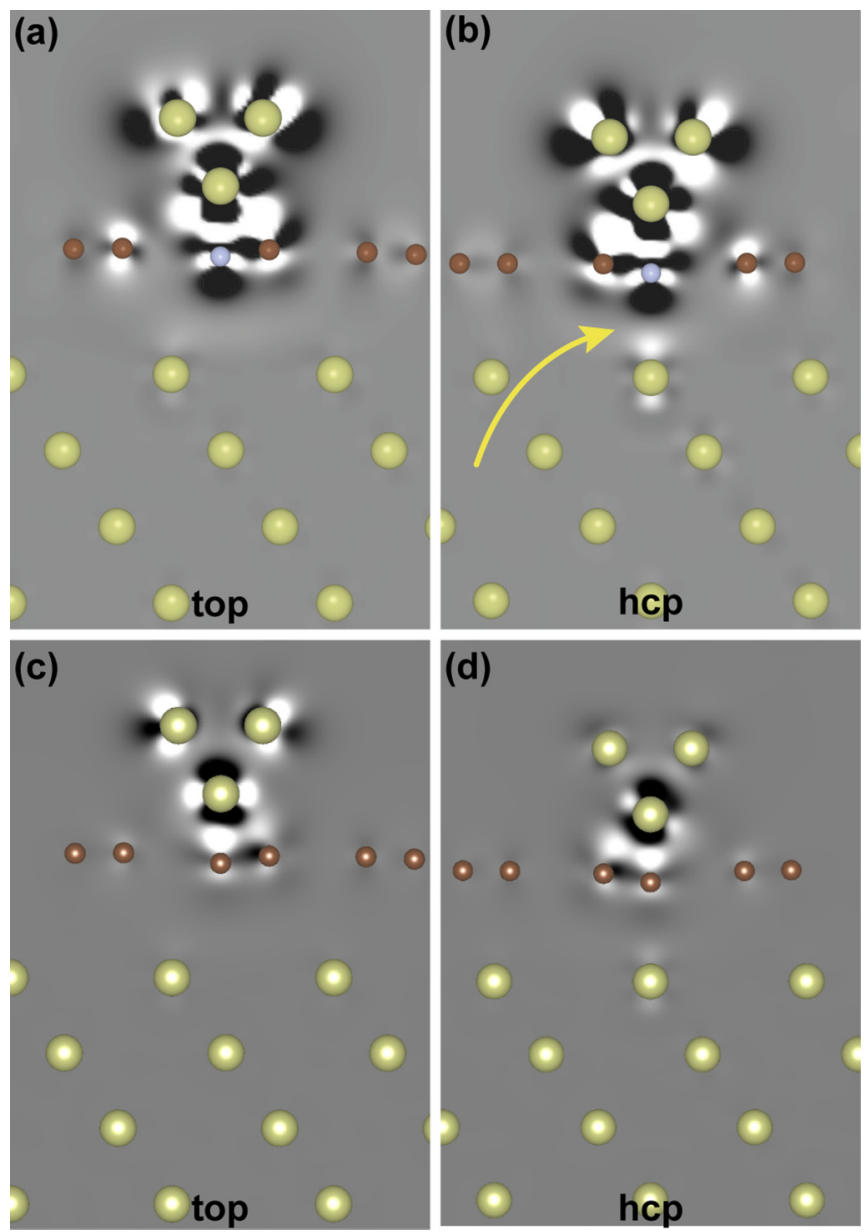

FIG. 8. Charge density difference $\Delta \rho=\rho_{\text {tip }, S}-\rho_{\text {tip }}-\rho_{S}$, plotted in $\left(e / \AA^{3}\right)$, for $\mathrm{N}$ at (a) top or (b) hcp sites, and for pristine graphene at (c) top and (d) hcp sites. $\rho_{S}$ is the charge density of the isolated (N-doped) graphene/Ir(111) system, $\rho_{\text {tip }}$ is the charge density of the isolated model tip, and $\rho_{\text {tip }, S}$ is the charge density of the overall system, with the tip at small distance from the surface. The color scale ranges from $-0.013 e / \AA^{3}$ (black; loss in electron density) to $0.013 e / \AA^{3}$ (white; gain in electron density), passing through zero (gray). The charge densities have been calculated after allowing the tip apex, the probed atom in the graphene layer, and its three nearest neighbors to relax. Ir, $\mathrm{C}$, and $\mathrm{N}$ atoms are marked in green, brown, and blue, respectively. The yellow arrow highlights the relatively stronger chemical bond which is formed between $\mathrm{N}$ and $\operatorname{Ir}(111)$ at the hcp site. 
(dashed blue). Because this difference is only observed for the calculation with substrate, this difference must be due to the interaction between the $\operatorname{Ir}(111)$ substrate and the N-dopant atom at the hcp site, an interaction that is absent between a $\mathrm{C}$ atom at an hcp site and the $\operatorname{Ir}(111)$ substrate.

Figure 8 shows the electron density difference maps of a side view of the tip (at $z=z_{\min }$ ), the $\mathrm{N}$-doped graphene or pristine graphene, and the Ir substrate. The yellow arrow in Fig. 8(b) points to the area between the $\mathrm{N}$ dopant at the hcp site and the Ir substrate. There is a significant redistribution of electron density difference in the area between the $\mathrm{N}$ atom in the hcp site and the underlying substrate. In contrast, such a redistribution is not observed for the $\mathrm{N}$ atom in a top site of the moiré lattice; see Fig. 8(a). The electron density difference maps of pristine graphene shown in Figs. 8(c) and 8(d) confirm our conclusions about the lack of interaction between the $\operatorname{Ir}(111)$ substrate and a $C$ atom in both the top and hcp sites.

\section{CONCLUSION}

In summary, we have explored the capabilities and performance of AFM for the identification and characterization of $\mathrm{N}$ dopants in graphene. Using chemical vapor deposition, we grew graphene on $\mathrm{Cu}(111)$ and $\operatorname{Ir}(111)$ surfaces using two different $\mathrm{N}$ containing precursor molecules. In all cases, clear signatures of $\mathrm{N}$ atoms incorporated in the lattice where observed in STM images. These were used as a benchmark for the AFM experiments. For both substrates, the $\Delta f(z)$ curves acquired above $\mathrm{N}$ and $\mathrm{C}$ located at similar positions with respect to the moiré lattice exhibit significant differences. In particular, the location of the minimum, $\Delta f_{\min }$, and $z_{\min }$ were found to contain information on the chemical nature of the atom above which the curve was acquired. The experimental findings are reproduced using DFT calculations. We expect the method described above to also be applicable to other dopants.

\section{ACKNOWLEDGMENTS}

Financial support from the Nederlandse Organisatie voor Wetenschappelijk Onderzoek (NWO; ECHO-Stip Grant No. 717.013.003 and a VIDI grant) is gratefully acknowledged.
[1] K. S. Novoselov, A. K. Geim, S. V. Morozov, D. Jiang, Y. Zhang, S. V. Dubonos, I. V. Grigorieva, and A. A. Firsov, Science 306, 666 (2004).

[2] T. Schiros, D. Nordlund, L. Pálová, D. Prezzi, L. Zhao, K. S. Kim, U. Wurstbauer, C. Gutiérrez, D. Delongchamp, C. Jaye, D. Fischer, H. Ogasawara, L. G. M. Pettersson, D. R. Reichman, P. Kim, M. S. Hybertsen, and A. N. Pasupathy, Nano Lett. 12, 4025 (2012).

[3] H. Wang, T. Maiyalagan, and X. Wang, ACS Catalysis 2, 781 (2012).

[4] R. R. Cloke, T. Marangoni, G. D. Nguyen, T. Joshi, D. J. Rizzo, C. Bronner, T. Cao, S. G. Louie, M. F. Crommie, and F. R. Fischer, J. Am. Chem. Soc. 137, 8872 (2015).

[5] S. Kawai, S. Saito, S. Osumi, S. Yamaguchi, A. S. Foster, P. Spijker, and E. Meyer, Nat. Commun. 6, 8098 (2015).

[6] S. Stankovich, D. A. Dikin, R. D. Piner, K. A. Kohlhaas, A. Kleinhammes, Y. Jia, Y. Wu, S. T. Nguyen, and R. S. Ruoff, Carbon 45, 1558 (2007).

[7] A. T. N'Diaye, M. Engler, C. Busse, D. Wall, N. Buckanie, F.-J. Meyer zu Heringdorf, R. van Gastel, B. Poelsema, and T. Michely, New J. Phys. 11, 023006 (2009).

[8] A. Deshpande, W. Bao, F. Miao, C. N. Lau, and B. J. LeRoy, Phys. Rev. B 79, 205411 (2009).

[9] L. Gao, J. R. Guest, and N. P. Guisinger, Nano Lett. 10, 3512 (2010).

[10] C. Busse, P. Lazić, R. Djemour, J. Coraux, T. Gerber, N. Atodiresei, V. Caciuc, R. Brako, A. T. N'Diaye, S. Blügel, J. Zegenhagen, and T. Michely, Phys. Rev. Lett. 107, 036101 (2011).

[11] R. Decker, Y. Wang, V. W. Brar, W. Regan, H.-Z. Tsai, Q. Wu, W. Gannett, A. Zettl, and M. F. Crommie, Nano Lett. 11, 2291 (2011).
[12] J. Xue, J. Sanchez-Yamagishi, D. Bulmash, P. Jacquod, A. Deshpande, K. Watanabe, T. Taniguchi, P. Jarillo-Herrero, and B. J. LeRoy, Nat. Mater. 10, 282 (2011).

[13] M. M. Ugeda, D. Fernández-Torre, I. Brihuega, P. Pou, A. J. Martínez-Galera, R. Pérez, and J. M. Gómez-Rodríguez, Phys. Rev. Lett. 107, 116803 (2011).

[14] H. Wang, Q. Wang, Y. Cheng, K. Li, Y. Yao, Q. Zhang, C. Dong, P. Wang, U. Schwingenschlögl, W. Yang, and X. X. Zhang, Nano Lett. 12, 141 (2012).

[15] M. P. Boneschanscher, J. Van Der Lit, Z. Sun, I. Swart, P. Liljeroth, and D. Vanmaekelbergh, ACS Nano 6, 10216 (2012).

[16] N. Néel, J. Kröger, L. Limot, T. Frederiksen, M. Brandbyge, and R. Berndt, Phys. Rev. Lett. 98, 065502 (2007).

[17] X. Li, X. Wang, L. Zhang, S. Lee, and H. Dai, Science 319, 1229 (2008).

[18] J. Cai, P. Ruffieux, R. Jaafar, M. Bieri, T. Braun, S. Blankenburg, M. Muoth, A. P. Seitsonen, M. Saleh, X. Feng, K. Müllen, and R. Fasel, Nature (London) 466, 470 (2010).

[19] S. J. Altenburg, J. Kröger, T. O. Wehling, B. Sachs, A. I. Lichtenstein, and R. Berndt, Phys. Rev. Lett. 108, 206805 (2012).

[20] Y. C. Chen, D. G. De Oteyza, Z. Pedramrazi, C. Chen, F. R. Fischer, and M. F. Crommie, ACS Nano 7, 6123 (2013).

[21] J. van der Lit, P. H. Jacobse, D. Vanmaekelbergh, and I. Swart, New J. Phys. 17, 053013 (2015).

[22] R. Lv, Q. Li, A. R. Botello-Méndez, T. Hayashi, B. Wang, A. Berkdemir, Q. Hao, A. L. Elías, R. Cruz-Silva, H. R. Gutiérrez, Y. A. Kim, H. Muramatsu, J. Zhu, M. Endo, H. Terrones, J.-C. Charlier, M. Pan, and M. Terrones, Sci. Rep. 2, 1 (2012).

[23] M. Telychko, P. Mutombo, M. Ondráček, P. Hapala, F. C. Bocquet, J. Kolorenč, M. Vondráček, P. Jelínek, and M. Švec, ACS Nano 8, 7318 (2014). 
[24] M. Telychko, P. Mutombo, P. Merino, P. Hapala, M. Ondráček, F. C. Bocquet, J. Sforzini, O. Stetsovych, M. Vondráček, P. Jelínek, and M. Švec, ACS Nano 9, 9180 (2015).

[25] A. Zabet-Khosousi, L. Zhao, L. Pálová, M. S. Hybertsen, D. R. Reichman, A. N. Pasupathy, and G. W. Flynn, J. Am. Chem. Soc. 136, 1391 (2014).

[26] K.-J. Kim, S. Yang, Y. Park, M. Lee, B. Kim, and H. Lee, J. Phys. Chem. C 117, 2129 (2013).

[27] H. Hölscher, W. Allers, U. D. Schwarz, A. Schwarz, and R. Wiesendanger, Phys. Rev. B 62, 6967 (2000).

[28] S. Hembacher, F. J. Giessibl, J. Mannhart, and C. F. Quate, Proc. Natl. Acad. Sci. USA 100, 12539 (2003).

[29] S. Hembacher, F. J. Giessibl, J. Mannhart, and C. F. Quate, Phys. Rev. Lett. 94, 056101 (2005).

[30] B. J. Albers, T. C. Schwendemann, M. Z. Baykara, N. Pilet, M. Liebmann, E. I. Altman, and U. D. Schwarz, Nat. Nanotechnol. 4, 307 (2009).

[31] M. Ondráček, P. Pou, V. Rozsíval, C. González, P. Jelínek, and R. Pérez, Phys. Rev. Lett. 106, 176101 (2011).

[32] Z. Sun, S. K. Hämäläinen, J. Sainio, J. Lahtinen, D. Vanmaekelbergh, and P. Liljeroth, Phys. Rev. B 83, 081415(R) (2011).

[33] E. N. Voloshina, E. Fertitta, A. Garhofer, F. Mittendorfer, M. Fonin, A. Thissen, and Y. S. Dedkov, Sci. Rep. 3, 1072 (2013).

[34] S. K. Hämäläinen, M. P. Boneschanscher, P. H. Jacobse, I. Swart, K. Pussi, W. Moritz, J. Lahtinen, P. Liljeroth, and J. Sainio, Phys. Rev. B 88, 201406 (2013).

[35] Y. Sugimoto, P. Pou, M. Abe, P. Jelinek, R. Pérez, S. Morita, and O. Custance, Nature (London) 446, 64 (2007).
[36] P. E. Blöchl, Phys. Rev. B 50, 17953 (1994).

[37] G. Kresse and J. Furthmüller, Phys. Rev. B 54, 11169 (1996).

[38] J. Lehtomäki, I. Makkonen, M. A. Caro, A. Harju, and O. LopezAcevedo, J. Chem. Phys. 141, 234102 (2014).

[39] J. P. Perdew, K. Burke, and M. Ernzerhof, Phys. Rev. Lett. 77, 3865 (1996).

[40] D. Vanderbilt, Phys. Rev. B 41, 7892 (1990).

[41] S. Grimme, Wiley Interdiscip. Rev.: Comput. Mol. Sci. 1, 211 (2011).

[42] S. Grimme, J. Antony, S. Ehrlich, and H. Krieg, J. Chem. Phys. 132, 154104 (2010).

[43] D. Z. Gao, J. Grenz, M. B. Watkins, F. Federici Canova, A. Schwarz, R. Wiesendanger, and A. L. Shluger, ACS Nano 8, 5339 (2014).

[44] F. J. Giessibl, Phys. Rev. B 56, 16010 (1997).

[45] L. Zhao, R. He, K. T. Rim, T. Schiros, K. S. Kim, H. Zhou, C. Gutierrez, S. P. Chockalingam, C. J. Arguello, L. Palova, D. Nordlund, M. S. Hybertsen, D. R. Reichman, T. F. Heinz, P. Kim, A. Pinczuk, G. W. Flynn, and A. N. Pasupathy, Science 333, 999 (2011).

[46] D. Wei, Y. Liu, Y. Wang, H. Zhang, L. Huang, and G. Yu, Nano Lett. 9, 1752 (2009).

[47] J. van der Lit, F. Di Cicco, P. Hapala, P. Jelinek, and I. Swart, Phys. Rev. Lett. 116, 096102 (2016).

[48] P. Hapala, M. Švec, O. Stetsovych, N. J. van der Heijden, M. Ondráček, J. van der Lit, P. Mutombo, I. Swart, and P. Jelínek, Nat. Commun. 7, 11560 (2016).

[49] M. P. Boneschanscher, S. K. Hämäläinen, P. Liljeroth, and I. Swart, ACS Nano 8, 3006 (2014). 